Taxonomía y sistemática

\title{
Helechos y licofitas del municipio de Cardonal, Hidalgo, México
}

\author{
Ferns and lycophytes of the Cardonal Municipality, Hidalgo, Mexico \\ Laura Guzmán-Cornejo, Andrés Sánchez-Morales y Leticia Pacheco* \\ Departamento de Biología, División de Ciencias Biológicas y de la Salud, Universidad Autónoma Metropolitana-Iztapalapa, Apartado postal 55-535, 09340 \\ Ciudad de México, D.F., México
}

Recibido el 24 de enero de 2014; aceptado el 12 de febrero de 2015

Disponible en Internet el 21 de agosto de 2015

\begin{abstract}
Resumen
Se realizó un estudio florístico de los helechos y licofitas del municipio de Cardonal, Hidalgo. Para ello, se revisaron las colecciones de varios herbarios, a fin de obtener una lista preliminar de helechos y licofitas del área de estudio. Paralelamente, se recolectó material botánico en 6 tipos de vegetación de febrero a abril y septiembre a octubre; los ejemplares fueron identificados, montados, etiquetados e incorporados a la colección del Herbario Metropolitano (UAMIZ). Se encontraron 63 taxones, de los cuales 9 corresponden a licofitas; Asplenium gentryi, Pleopeltis platylepis y Selaginella ribae, son registros nuevos para Hidalgo. Con los datos obtenidos, se elaboró una matriz de ausencia-presencia de las especies por tipos de vegetación y se aplicó un análisis de correspondencia y otro de agrupamiento para determinar la similitud de las especies entre los sitios muestreados. El análisis estadístico mostró que los bosques de Pinus, Quercus y tropical caducifolio presentan una composición florística de helechos y licofitas independiente entre sí, mientras que en los bosques de Juniperus, espinoso y matorral xerófilo es similar. La diversidad de condiciones ambientales presentes en Cardonal le confiere una riqueza florística comparable o mayor, en cuanto al número de especies, a la de otras zonas protegidas (3) del estado de Hidalgo.

Derechos Reservados ( 2015 Universidad Nacional Autónoma de México, Instituto de Biología. Este es un artículo de acceso abierto distribuido bajo los términos de la Licencia Creative Commons CC BY-NC-ND 4.0.
\end{abstract}

Palabras clave: Diversidad; Endémico; Flora; Nuevos registros; Pteridofitas

\section{Abstract}

A floristic research of ferns and lycophytes was made at Cardonal Municipality, Hidalgo. For this purpose, several samples of different herbaria were revised in order to get a preliminary list of the ferns and lycophytes of this zone. At the same time, botanical samples were collected in 6 different types of vegetation from February to April and September to October; every sample was identified, set, labeled and incorporated to the collection of the Metropolitan Herbarium (UAMIZ). There were found 63 taxa, of which 9 correspond to lycophytes; Asplenium gentryi, Pleopeltis platylepis and Selaginella ribae, are new records for Hidalgo. With the obtained data, an absence-presence matrix of species found in the different types of vegetation was made, it was analized through correspondence and grouping analyses to determine the species similarity found among sampled sites. The statistical analyses showed that Pinus, Quercus and tropical deciduous forests have a floristic composition of ferns and lycophytes independent between them, while the Juniperus forest, thorny and tropical scrubs, present a similar composition. The diversity of environmental conditions found at Cardonal confers a floristic richness greater or equal-in number of species-, to the one present in protected zones (3) of the state of Hidalgo.

All Rights Reserved $\odot 2015$ Universidad Nacional Autónoma de México, Instituto de Biología. This is an open access item distributed under the Creative Commons CC License BY-NC-ND 4.0.

Keywords: Diversity; Endemic; Flora; New records; Pteridophytes

\footnotetext{
* Autor para correspondencia.

Correo electrónico: pacheco@xanum.uam.mx (L. Pacheco).

La revisión por pares es responsabilidad de la Universidad Nacional Autónoma de México.
} 


\section{Introducción}

México posee gran diversidad de helechos y licofitas, Mickel y Smith (2004) citan 1,008 especies y 16 variedades o subespecies distribuidas en diferentes hábitats, lo cual representa el $3.92 \%$ si comparamos con el número de plantas vasculares de México, calculada en 25,701 especies (Espejo-Serna, López-Ferrari y Salgado-Ugarte, 2004). La mayor riqueza y diversidad de helechos y licofitas se encuentra en las zonas tropicales, siendo los bosques mesófilos los que cuentan con el mayor número de especies, seguidos por los bosques tropicales perennifolios y subperennifolios, los bosques de pino-encino, los bosques tropicales caducifolios, la vegetación xérica y la acuática (Riba, 1998). Después de Oaxaca, Chiapas, Veracruz y Puebla, Hidalgo es uno de los estados con mayor riqueza de helechos y licopodios (Mickel y Smith, 2004). Actualmente el estado no cuenta con un listado florístico detallado y completo; sin embargo, Sánchez-Mejorada y Chávez (1951) citaron, en su estudio de la barranca de Omitlán, los primeros registros para la entidad.

Recientemente se han generado algunos listados florísticos, principalmente de la región de la huasteca hidalguense, así como en áreas protegidas como el parque nacional El Chico (Serrano, 2010), el parque nacional Los Mármoles (RamírezCruz, Sánchez-González y Tejero-Díez, 2009), la Reserva de la Biosfera Barranca de Metztitlán (Conanp, 2003) o también en algunos municipios como Zacualtipán de Ángeles (Alcántara-Ayala y Luna-Vega, 2001; Pérez-Paredes, SánchezGonzález y Tejero-Díez, 2012), Calnali (Pérez-Cervantes, 2009), Tlanchinol (Álvarez-Zúñiga, Sánchez-González, LópezMata y Tejero-Díez, 2012), Tenango de Doria (Alcántara-Ayala y Luna-Vega, 1997; Pérez-Paredes, Serrano, Álvarez y SánchezGonzález, 2011) y en parte de los municipios de Eloxochitlán (Alcántara-Ayala y Luna-Vega, 2001) y Lolotla (Ponce-Vargas, Luna-Vega, Alcántara-Ayala y Ruiz-Jiménez, 2006); el listado de las epífitas vasculares de Hidalgo (Ceja-Romero et al., 2010) incluye una parte de los helechos y licofitas, pero no citan ningún taxón para Cardonal. Sin embargo, gran parte de las zonas semiáridas del estado aún no cuentan con este tipo de estudios. Por lo que el objetivo de este trabajo es dar a conocer la riqueza de helechos y licofitas de Cardonal, contribuyendo con ello al conocimiento florístico del municipio, del estado de Hidalgo y de la República Mexicana.

\section{Materiales y métodos}

El municipio de Cardonal tiene una extensión territorial de $462.60 \mathrm{~km}^{2}$, lo que representa $2.21 \%$ de los $20,905.12 \mathrm{~km}^{2}$ con que cuenta el estado de Hidalgo. Se ubica entre las siguientes coordenadas: $20^{\circ} 25^{\prime}-20^{\circ} 47^{\prime} \mathrm{N}, 98^{\circ} 55^{\prime}-99^{\circ} 11^{\prime} \mathrm{O}$, a una altitud entre 900 y 2,900 msnm (Inegi, 2009). Colinda al norte con los municipios de Nicolás Flores y Tlahuiltepa; al sur con Santiago de Anaya e Ixmiquilpan; al este con Eloxochitlán, Metztitlán, Tlahuiltepa y Santiago de Anaya; al oeste con Ixmiquilpan y Nicolás Flores (figura 1). Fisiográficamente, el 96\% del municipio ocupa territorio de la Sierra Madre Oriental y el
4\% restante, del Eje Neovolcánico Transmexicano. Pertenece a la región hidrológica del Pánuco y a la cuenca del río Moctezuma. Los climas que se citan para el municipio son el templado subhúmedo con lluvias en verano, el semiseco templado, el semiseco semicálido y el seco semicálido. Presenta un intervalo de precipitación que va de 300 a $1,100 \mathrm{~mm}$, y uno de temperatura entre 12 y $22^{\circ} \mathrm{C}$. La vegetación que menciona el Inegi (2009) para la zona de estudio se compone de bosques que ocupan $29 \%$ del municipio, matorral xerófilo que cubre 18\%, áreas utilizadas para la agricultura 33\% y los pastizales ocupan el 20\% restante del territorio.

La recolección del material biológico se realizó a partir de febrero de 2009 a septiembre de 2011, visitando en varias ocasiones cada sitio, tanto en época de lluvias: septiembre y octubre, como de sequía: febrero a abril. Los sitios de recolección fueron elegidos por sus características topográficas, climáticas, edáficas y vegetación dominante (figura 1), recolectando de 3-5 duplicados por especie y localidad, de acuerdo con lo propuesto por Lorea y Riba (1990).

Los ejemplares fueron determinados a nivel de género y especie utilizando los trabajos de Mickel y Smith (2004), para algunos géneros se actualizó la nomenclatura y taxonomía de acuerdo con Fay-Wei, Pryer y Windham (2012), Grusz y Windham (2013) y Smith y Tejero-Díez (2014). La asignación de familias se hizo de acuerdo con lo propuesto por Smith et al. (2006). Los ejemplares identificados y debidamente etiquetados se montaron para ser incorporados al Herbario Metropolitano Dr. Ramón Riba y Nava Esparza (UAMIZ).

Paralelamente se hizo una revisión de las colecciones de los herbarios de la Escuela Nacional de Ciencias Biológicas del IPN (ENCB), de la Universidad Autónoma del Estado de Hidalgo (HGOM), Herbario Nacional del Instituto de Biología, UNAM (MEXU) y de la Universidad Autónoma Metropolitana (UAMIZ), con el fin de obtener una lista preliminar de helechos y licofitas de la zona de estudio. Con la información obtenida se elaboró un listado florístico de las familias, géneros y especies encontrados en el municipio en relación con el tipo de vegetación donde fueron recolectados.

Con la lista de especies obtenida se elaboró una matriz de presencia-ausencia, considerando a las especies como variables y a los tipos de vegetación como los componentes. Para analizar las afinidades en la composición florística de helechos y licopodios de los distintos tipos de vegetación del municipio, se realizó un análisis de correspondencia, mismo que fue reforzado por 2 análisis de agrupamiento, uno usando varianza mínima de Ward y otro utilizando grupos promedio. Todos se realizaron en el paquete estadístico NCSS.

\section{Resultados}

Como resultado del trabajo de campo se recolectaron 141 ejemplares de helechos y licopodios. En la revisión de herbarios solo se encontraron 7 ejemplares de colectores ajenos a este trabajo; de estos, 4 estaban depositados en MEXU y los otros 3 en UAMIZ (tabla 1). La revisión de ejemplares de herbario permitió determinar que las zonas semiáridas de Hidalgo han sido poco recolectadas, siendo la Huasteca y las zonas templadas las 
Tabla 1

Lista de especies, colectores y herbarios consultados.

\begin{tabular}{|c|c|c|}
\hline Especie & Colector y número & Herbario \\
\hline Adiantum braunii Mett. ex Kuhn & L. Guzmán-Cornejo et al., 163 & UAMIZ \\
\hline Adiantum capillus-veneris $\mathrm{L}$. & L. Guzmán-Cornejo et al. 2, 37, 132 & UAMIZ \\
\hline Adiantum poiretii Wikstr. & L. Guzmán-Cornejo et al. 89 & UAMIZ \\
\hline Anemia adiantifolia (L.) Sw. & L. Guzmán-Cornejo et al. 125 & UAMIZ \\
\hline Anemia mexicana Klotzsch var. mexicana & L. Guzmán-Cornejo et al. 136 & UAMIZ \\
\hline Argyrochosma formosa (Liebm.) Windham & L. Guzmán-Cornejo et al. 127, 128, 193 & UAMIZ \\
\hline Asplenium gentryi A. R. Sm." & L. Guzmán-Cornejo et al. 91, 115, 120, 130, 187 & UAMIZ \\
\hline Asplenium monanthes $\mathrm{L}$. & L. Guzmán-Cornejo et al. $35,38,102,141$ & UAMIZ \\
\hline Asplenium palmeri Maxon & L. Guzmán-Cornejo et al. 79 & UAMIZ \\
\hline Asplenium resiliens Kunze & L. Guzmán-Cornejo et al. 173 & UAMIZ \\
\hline $\begin{array}{l}\text { Astrolepis crassifolia (T. Moore y Houlston) D. M. Benham } \\
\text { y Windham }\end{array}$ & L. Guzmán-Cornejo et al. 85 & UAMIZ \\
\hline Astrolepis integerrima (Hook.) D. M. Benham y Windham & L. Guzmán-Cornejo et al. 84, 131, 180 & UAMIZ \\
\hline Astrolepis sinuata (Lag. ex Sw.) D. M. Benham y Windham & L. Guzmán-Cornejo et al. 30, 166, 170, 175, 189 & UAMIZ \\
\hline Botrychium virginianum (L.) Sw. & L. Guzmán-Cornejo et al. 97, 146 & UAMIZ \\
\hline Cystopteris fragilis (L.) Bernh. & L. Guzmán-Cornejo et al. 92, 144, 157 & UAMIZ \\
\hline \multirow[t]{2}{*}{ Cheilanthes bonariensis (Willd.) Proctor } & J. Ceja y A. Mendoza R. 1546 & UAMIZ \\
\hline & L. Guzmán-Cornejo et al. 44, 45, 99, 108, 148, 171 & UAMIZ \\
\hline Cheilanthes cucullans Fée & L. Guzmán-Cornejo et al. 109, 112, 192 & UAMIZ \\
\hline Cheilanthes microphylla (Sw.) Sw. & L. Guzmán-Cornejo et al. 1 & UAMIZ \\
\hline Cheilanthes notholaenoides (Desv.) Maxon ex Weath. & $\begin{array}{l}\text { L. Guzmán-Cornejo et al. } 33,87,88,117,123,129,143 \text {, } \\
156,169,176,182,186\end{array}$ & UAMIZ \\
\hline Cheilanthes spiculata Mickel & L. Guzmán-Cornejo et al. 158 & UAMIZ \\
\hline $\begin{array}{l}\text { Equisetum hyemale A. Braun ex Engelm. L. var. affine } \\
\text { (Engelm.) A. A. Eaton }\end{array}$ & L. Guzmán-Cornejo et al. 96,135 & UAMIZ \\
\hline Elaphoglossum petiolatum (Sw.) Urb. & L. Guzmán-Cornejo et al. 40 & UAMIZ \\
\hline Gaga purpusii (T. Reeves) Fay-Wey Li y Windham & L. Guzmán-Cornejo et al. 119 & UAMIZ \\
\hline Llavea cordifolia Lag. & L. Guzmán-Cornejo et al. 101 & UAMIZ \\
\hline Mildella fallax (M. Martens y Galeotti) Nesom & L. Guzmán-Cornejo et al. 34, 86, 142, 159, 178 & UAMIZ \\
\hline Myriopteris windhamii Grusz & L. Guzmán-Cornejo et al. 106 & UAMIZ \\
\hline Notholaena aschenborniana Klotzsch & L. Guzmán-Cornejo et al. 105, 183, 190 & UAMIZ \\
\hline Notholaena galeotti Fée & L. Guzmán-Cornejo et al. $110,111,167$ & UAMIZ \\
\hline \multirow[t]{2}{*}{ Notholaena jacalensis Pray } & F. González-Medrano et al. s.n. (MEXU 298516) & MEXU \\
\hline & L. Guzmán-Cornejo et al. 31, 181 & UAMIZ \\
\hline Notholaena sulphurea (Cav.) J. Sm. & L. Guzmán-Cornejo et al. 5, 32, 107, 165 & UAMIZ \\
\hline Pecluma plumula (Humb. y Bonpl. ex Willd.) M. G. Price & L. Guzmán-Cornejo et al. 121 & UAMIZ \\
\hline Pellaea cordifolia (Sessé y Moç.) A. R. Sm. & L. Guzmán-Cornejo et al. 100, 168, 174 & UAMIZ \\
\hline Pellaea sagittata (Cav.) Link & L. Guzmán-Cornejo et al. 160 & UAMIZ \\
\hline Phlebodium areolatum (Humb. y Bonpl. ex Willd.) J. Sm. & L. Guzmán-Cornejo et al. 137 & UAMIZ \\
\hline Pleopeltis crassinervata (Fée) T. Moore & L. Guzmán-Cornejo et al. 41 & UAMIZ \\
\hline Pleopeltis furfuracea (Schltdl. y Cham.) A.R. Sm. y Tejero & L. Guzmán-Cornejo et al. 126 & UAMIZ \\
\hline Pleopeltis guttata (Maxon) E. G. Andrews y Windham & L. Guzmán-Cornejo et al. 83 & UAMIZ \\
\hline Pleopeltis madrensis (J. Sm.) A.R. Sm. y Tejero & L. Guzmán-Cornejo et al. 153 & UAMIZ \\
\hline Pleopeltis mexicana (Fée) Mickel y Beitel & L. Guzmán-Cornejo et al. 152 & UAMIZ \\
\hline Pleopeltis platylepis (Mett. ex Kuhn.) A.R. Sm. y Tejero * & L. Guzmán-Cornejo et al. 82, 149 & UAMIZ \\
\hline Pleopeltis plebeia (Schltdl. y Cham.) A.R. Sm. y Tejero & L. Guzmán-Cornejo et al. 118 & UAMIZ \\
\hline $\begin{array}{l}\text { Pleopeltis polylepis (Roemer ex Kunze) T. Moore var. } \\
\quad \text { polylepis }\end{array}$ & L. Guzmán-Cornejo et al. $80,81,114,151,154,185,188$ & UAMIZ \\
\hline $\begin{array}{l}\text { Pleopeltis polypodioides (L.) E. G. Andrews y Windham } \\
\text { var. acicularis (Weath.) E. G. Andrews y Windham }\end{array}$ & L. Guzmán-Cornejo et al. 36, 116, 184 & UAMIZ \\
\hline $\begin{array}{l}\text { Pleopeltis thyssanolepis (A. Braun ex Klotzsch) E. G. } \\
\text { Andrews y Windham }\end{array}$ & L. Guzmán-Cornejo et al. 42, 95, 122, 196 & UAMIZ \\
\hline Polypodium martensii Mett. & L. Guzmán-Cornejo et al. 103, 150, 164 & UAMIZ \\
\hline Polypodium plesiosorum Kunze & L. Guzmán-Cornejo et al. 104,147 & UAMIZ \\
\hline Polypodium subpetiolatum Hook & L. Guzmán-Cornejo et al. 162 & UAMIZ \\
\hline $\begin{array}{l}\text { Pteridium aquilinum (L.) Kuhn var. feei (W. Schaffn. ex } \\
\text { Fée) Maxon ex Yunck. }\end{array}$ & L. Guzmán-Cornejo et al. 139 & UAMIZ \\
\hline Pteris cretica $\mathrm{L}$. & L. Guzmán-Cornejo et al. 98 & UAMIZ \\
\hline Selaginella extensa Underw. & J. Ceja y A. Mendoza R. 1548 & UAMIZ \\
\hline \multirow[t]{3}{*}{ Selaginella lepidophylla (Hook. y Grev.) Spring } & P. Hiriart y G. Ortiz 12 & MEXU \\
\hline & H. Sánchez-Mejorada, 2641 & MEXU \\
\hline & L. Guzmán-Cornejo et al. 77, 133, 134, 155, 172, 177 & UAMIZ \\
\hline Selaginella pallescens (C. Presl) Spring & J. Ceja y A. Mendoza R. 1547 & UAMIZ \\
\hline Selaginella pilifera A. Braun & L. Guzmán-Cornejo et al. 78 & UAMIZ \\
\hline
\end{tabular}


Tabla 1 (continuación)

\begin{tabular}{lll}
\hline Especie & Colector y número & Herbario \\
\hline Selaginella pulcherrima Liebm. ex E. Fourn. & L. Guzmán-Cornejo et al. 43, 124 & UAMIZ \\
$\begin{array}{l}\text { Selaginella reflexa Underw. } \\
\text { Selaginella ribae Valdespino* }\end{array}$ & C. J. Rothfels et al. 3005 & MEXU \\
Selaginella sellowii Hieron. & L. Guzmán-Cornejo et al. 179, 195 & UAMIZ \\
Selaginella wrightii Hieron. & L. Guzmán-Cornejo et al. 194 & UAMIZ \\
Tectaria heracleifolia (Willd.) Underw. & L. Guzmán-Cornejo et al. 113 & UAMIZ \\
Thelypteris kunthii (Desv.) C.V. Morton & L. Guzmán-Cornejo et al. 4 & UAMIZ \\
Thelypteris oligocarpa (Humb. y Bonpl. ex Willd.) Ching & L. Guzmán-Cornejo et al. 3, 90, 93, 138, 140 & UAMIZ \\
Thelypteris pilosa (M. Martens y Galeotti) Crawford & L. Guzmán-Cornejo et al. 39 & UAMIZ \\
Woodsia mollis (Kaulf.) J. Sm. & L. Guzmán-Cornejo et al. 94, 191 & UAMIZ \\
\hline
\end{tabular}

\footnotetext{
* Nuevos registros para Hidalgo.
}

que más visitan los colectores, principalmente el municipio de Molango. Del total de plantas examinadas la mayoría presentan hábito terrestre $(80.9 \%)$, otras son epipétricas $(9.5 \%)$ y pocas fueron exclusivamente epífitas $(3.2 \%)$, ya que algunas pueden ser epífitas y/o epipétricas (5.4\%) (tabla 2).

Se registraron 63 taxones agrupados en 26 géneros y 12 familias, de las cuales 9 especies corresponden a la familia Selaginellaceae. Las familias mejor representadas en cuanto a géneros fueron Pteridaceae con 11 géneros (42.3\%) y Polypodiaceae con 4 taxones (15.3\%); el mayor número de especies pertenecen a Pleopeltis, Selaginella y Cheilanthes con 10, 9 y 5 , respectivamente (tabla 3). Las especies más frecuentes en el municipio fueron Cheilanthes notholaenoides (Desv.) Maxon ex Weath., Cheilanthes bonariensis (Willd.) Proctor, Thelypteris kunthii (Desv.) C. V. Morton y Pleopeltis polylepis (Roemer ex Kunze) T. Moore var. polylepis, encontrándose la primera presente en 5 tipos de vegetación y las demás en 4 (tabla 2).

Los tipos de vegetación presentes en el municipio fueron: bosque de pino (BP), bosque de Quercus (BQ), bosque de Juniperus (BJ), matorral xerófilo (MX), bosque espinoso (BE) y bosque tropical caducifolio (BTC) (figura 1; tabla 2) según Rzedowski (1978). El tipo de vegetación con mayor número de especies de helechos y licofitas fue el bosque de Quercus con 36 taxones (figura 2; tabla 4). Tres especies constituyen registros nuevos para el estado de Hidalgo: Asplenium gentryi A. R. Sm., Pleopeltis platylepis (Mett. ex Kuhn) A. R. Sm. et Tejero y Selaginella ribae Valdespino. De los 63 taxones recolectados en el municipio de Cardonal, 9 son endémicos de México: Asplenium gentryi, Cheilanthes spiculata Mickel, Myriopteris windhamii Grusz, Gaga purpusii (T. Reeves) Fay-Wei Li et Windham,

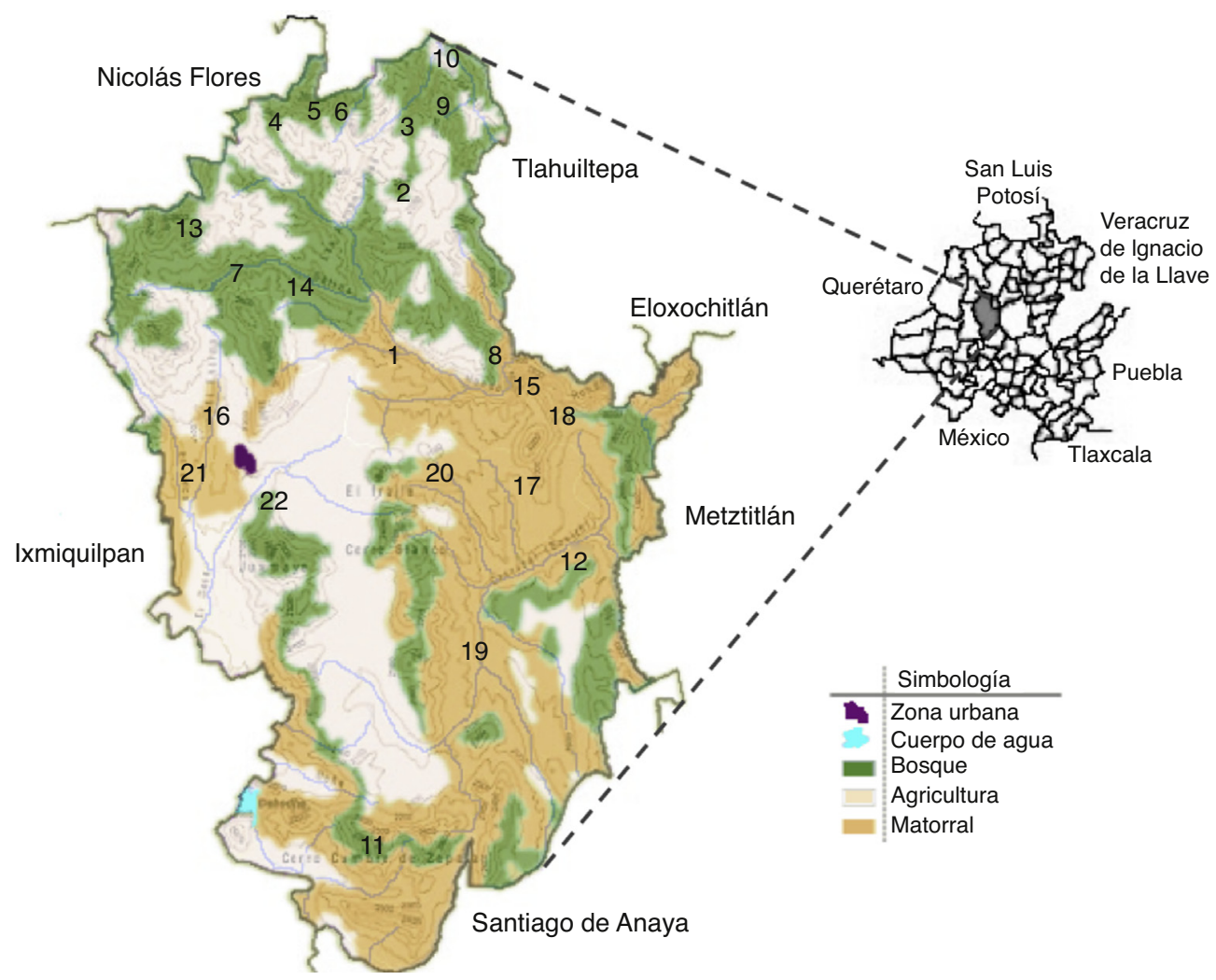

Figura 1. Localización del municipio de Cardonal, estado de Hidalgo, México. Los números corresponden a las localidades visitadas. Modificado de Inegi (2009). 
Tabla 2

Listado de helechos y licofitas del municipio de Cardonal, se indica el hábito, la localidad, así como los tipos de vegetación.

\begin{tabular}{|c|c|c|c|c|c|c|c|c|}
\hline \multirow[t]{2}{*}{ Especie } & \multirow[t]{2}{*}{ Hábito } & \multirow[t]{2}{*}{ Localidad } & \multicolumn{6}{|c|}{ Tipos de vegetación } \\
\hline & & & $\mathrm{BP}$ & $\mathrm{BJ}$ & BQ & $\mathrm{BE}$ & BTC & MX \\
\hline \multicolumn{9}{|l|}{ Anemiaceae } \\
\hline Anemia adiantifolia (L.) Sw. & Terrestre & 9 & $\mathrm{X}$ & & & & & \\
\hline Anemia mexicana Klotzsch var. mexicana & Terrestre & 9 & $\mathrm{X}$ & & & & & \\
\hline \multicolumn{9}{|l|}{ Aspleniaceae } \\
\hline Asplenium gentryi A.R. Sm.* & Terrestre & $7,9,11$ & $\mathrm{X}$ & $\mathrm{X}$ & & & & $\mathrm{X}$ \\
\hline Asplenium monanthes L. & Terrestre & $2,5,6$ & & & $\mathrm{X}$ & & & \\
\hline Asplenium palmeri Maxon & Terrestre & 7 & & & & & & $\mathrm{X}$ \\
\hline Asplenium resiliens Kunze & Terrestre & 7 & & & & & & $\mathrm{X}$ \\
\hline \multicolumn{9}{|l|}{ Dennstaedtiaceae } \\
\hline $\begin{array}{l}\text { Pteridium. aquilinum (L.) Kuhn var. feei (W. } \\
\text { Schaffn. ex Fée) Maxon ex Yunck. }\end{array}$ & Terrestre & 9 & $\mathrm{X}$ & & & & & \\
\hline \multicolumn{9}{|l|}{ Dryopteridaceae } \\
\hline Elaphoglossum petiolatum (Sw.) Urb. & Terrestre & 2 & & & $\mathrm{X}$ & & & \\
\hline \multicolumn{9}{|l|}{ Equisetaceae } \\
\hline $\begin{array}{l}\text { Equisetum hyemale A. Braun ex Engelm. L. var. } \\
\text { affine (Engelm.) A. A. Eaton }\end{array}$ & Terrestre & 6,10 & & & $\mathrm{X}$ & & & $\mathrm{X}$ \\
\hline \multicolumn{9}{|l|}{ Ophioglossaceae } \\
\hline Botrychium virginianum (L.) Sw. & Terrestre & 6 & & & $\mathrm{X}$ & & & \\
\hline \multicolumn{9}{|l|}{ Polypodiaceae } \\
\hline $\begin{array}{l}\text { Pecluma plumula (Humb. y Bonpl. ex Willd.) M.G. } \\
\text { Price }\end{array}$ & Epipétrico & 9 & $\mathrm{X}$ & & & & & \\
\hline $\begin{array}{l}\text { Phlebodium areolatum (Humb. y Bonpl. ex Willd.) } \\
\text { J. Sm. }\end{array}$ & Terrestre & 9 & $\mathrm{X}$ & & & & & \\
\hline Pleopeltis crassinervata (Fée) T. Moore & Epífito & 2 & & & $\mathrm{X}$ & & & \\
\hline $\begin{array}{l}\text { Pleopeltis furfuracea (Schltdl. y Cham.) A.R. Sm. y } \\
\text { Tejero }\end{array}$ & Epipétrico & 9 & $\mathrm{X}$ & & & & & \\
\hline $\begin{array}{l}\text { Pleopeltis guttata (Maxon) E. G. Andrews y } \\
\text { Windham }\end{array}$ & Terrestre & 5 & & & $\mathrm{X}$ & & & \\
\hline Pleopeltis madrensis (J. Sm.) A.R. Sm. y Tejero & Terrestre & 5 & & & $\mathrm{X}$ & & & \\
\hline Pleopeltis mexicana (Fée) Mickel y Beitel & Terrestre & 6 & & & $\mathrm{X}$ & & & \\
\hline $\begin{array}{l}\text { Pleopeltis platylepis (Mett. ex Kuhn.) A.R. Sm. y } \\
\text { Tejero* }\end{array}$ & Epífito, Epipétrico & 6 & & & $\mathrm{X}$ & & & \\
\hline $\begin{array}{l}\text { Pleopeltis plebeia (Schltdl. y Cham.) A.R. Sm. y } \\
\text { Tejero }\end{array}$ & Terrestre & 9 & $\mathrm{X}$ & & & & & \\
\hline $\begin{array}{l}\text { Pleopeltis polylepis (Roemer ex Kunze) T. Moore } \\
\text { var. polylepis }\end{array}$ & Epipétrico, Epífito & $5,6,7,9,11$ & $\mathrm{X}$ & $\mathrm{X}$ & $\mathrm{x}$ & & & $\mathrm{X}$ \\
\hline $\begin{array}{l}\text { Pleopeltis polypodioides (L.) E. G. Andrews y } \\
\text { Windham var. acicularis (Weath.) E. G. Andrews } \\
\text { y Windham }\end{array}$ & Epipétrico & $2,9,11$ & $\mathrm{X}$ & $\mathrm{X}$ & $\mathrm{X}$ & & & \\
\hline $\begin{array}{l}\text { Pleopeltis thyssanolepis (A. Braun ex Klotzsch) E. } \\
\text { G. Andrews y Windham }\end{array}$ & Epífito, Epipétrico & $1,2,5,9$ & $\mathrm{X}$ & & $\mathrm{X}$ & & & $\mathrm{X}$ \\
\hline Polypodium martensii Mett. & Epífito & 5,6 & & & $\mathrm{X}$ & & & \\
\hline Polypodium plesiosorum Kunze & Epífito, Epipétrico & 6 & & & $\mathrm{X}$ & & & \\
\hline Polypodium subpetiolatum Hook & Terrestre & 5 & & & $\mathrm{X}$ & & & \\
\hline \multicolumn{9}{|l|}{ Pteridaceae } \\
\hline Adiantum braunii Mett. ex Kuhn & Terrestre & 5 & & & $\mathrm{X}$ & & & \\
\hline Adiantum capillus-veneris $\mathrm{L}$. & Terrestre & $2,12,15$ & & & $\mathrm{X}$ & & $\mathrm{X}$ & $\mathrm{X}$ \\
\hline Adiantum poiretii Wikstr. & Terrestre & 5 & & & $\mathrm{X}$ & & & \\
\hline Argyrochosma formosa (Liebm.) Windham & Terrestre & 9,11 & $\mathrm{X}$ & $\mathrm{X}$ & $\mathrm{X}$ & & & \\
\hline $\begin{array}{l}\text { Astrolepis crassifolia (T. Moore y Houlston) D. M. } \\
\text { Benham y Windham }\end{array}$ & Terrestre & 5 & & & $\mathrm{X}$ & & & \\
\hline $\begin{array}{l}\text { Astrolepis integerrima (Hook.) D. M. Benham y } \\
\text { Windham }\end{array}$ & Terrestre & $1,5,11$ & & $\mathrm{X}$ & $\mathrm{X}$ & & & $\mathrm{X}$ \\
\hline $\begin{array}{l}\text { Astrolepis sinuata (Lag. ex Sw.) D. M. Benham y } \\
\text { Windham }\end{array}$ & Terrestre & $1,7,11,13$ & & $\mathrm{X}$ & & $\mathrm{X}$ & & $\mathrm{X}$ \\
\hline Cheilanthes bonariensis (Willd.)Proctor & Terrestre & $3,4,5,6,8,13,16$ & $\mathrm{X}$ & & $\mathrm{X}$ & $\mathrm{X}$ & & $\mathrm{X}$ \\
\hline Cheilanthes cucullans Fée & Terrestre & $8,9,11$ & $\mathrm{X}$ & $\mathrm{X}$ & & & & $\mathrm{X}$ \\
\hline Cheilanthes microphylla (Sw.) Sw. & Terrestre & 8 & & & & & & $\mathrm{X}$ \\
\hline $\begin{array}{l}\text { Cheilanthes notholaenoides (Desv.) Maxon ex } \\
\text { Weath. }\end{array}$ & Terrestre & $2,5,6,7,9,11,12,13$ & $\mathrm{X}$ & $\mathrm{X}$ & $\mathrm{X}$ & $\mathrm{X}$ & & $\mathrm{X}$ \\
\hline Cheilanthes spiculata Mickel & Terrestre & 8 & & & $\mathrm{X}$ & & & \\
\hline Gaga purpusii (T. Reeves) Fay-Wey Li y Windham & Terrestre & 5 & & & $\mathrm{X}$ & & & \\
\hline
\end{tabular}


Tabla 2 (continuación)

\begin{tabular}{|c|c|c|c|c|c|c|c|c|}
\hline \multirow[t]{2}{*}{ Especie } & \multirow[t]{2}{*}{ Hábito } & \multirow[t]{2}{*}{ Localidad } & \multicolumn{6}{|c|}{ Tipos de vegetación } \\
\hline & & & $\mathrm{BP}$ & BJ & BQ & $\mathrm{BE}$ & BTC & MX \\
\hline Llavea cordifolia Lag. & Terrestre & 5 & & & $\mathrm{X}$ & & & \\
\hline Myriopteris windhamii Grusz & Terrestre & 9 & & & & & & $\mathrm{X}$ \\
\hline Notholaena aschenborniana Klotzsch & Terrestre & $8,11,12$ & & $\mathrm{X}$ & & & & $\mathrm{X}$ \\
\hline Notholaena galeotti Fée & Terrestre & $8,9,13$ & $\mathrm{X}$ & & & $\mathrm{X}$ & & $\mathrm{X}$ \\
\hline Pellaea cordifolia (Sessé y Moç.) A.R. Sm. & Terrestre & 7,13 & & & & $\mathrm{X}$ & & $\mathrm{X}$ \\
\hline Pellaea sagittata (Cav.) Link & Terrestre & 5 & & & $\mathrm{X}$ & & & \\
\hline Pteris cretica $\mathrm{L}$. & Terrestre & 5 & & & $\mathrm{X}$ & & & \\
\hline Selaginellaceae & & & & & & & & \\
\hline Selaginella extensa Underw. & Epipétrico & 16 & & & & & & $\mathrm{X}$ \\
\hline Selaginella lepidophylla (Hook. y Grev.) Spring & Terrestre & $5,7,12,14,18$ & $\mathrm{X}$ & & $\mathrm{X}$ & & & $\mathrm{X}$ \\
\hline Selaginella sellowii Hieron. & Terrestre & 11 & $\mathrm{X}$ & & & & & \\
\hline $\begin{array}{l}\text { Selaginella wrightii Hieron. } \\
\text { Tectariaceae }\end{array}$ & Epipétrico & 9 & $\mathrm{X}$ & & & & & \\
\hline $\begin{array}{l}\text { Tectaria heracleifolia (Willd.) Underw. } \\
\text { Thelypteridaceae }\end{array}$ & Terrestre & 15 & & & & & $\mathrm{X}$ & \\
\hline Thelypteris kunthii (Desv.) C.V. Morton & Terrestre & $5,7,10,11,15$ & & $\mathrm{X}$ & $\mathrm{X}$ & & $\mathrm{X}$ & $\mathrm{X}$ \\
\hline $\begin{array}{l}\text { Thelypteris oligocarpa (Humb. y Bonpl. ex Willd.) } \\
\text { Ching }\end{array}$ & Terrestre & 2 & & & $\mathrm{X}$ & & & \\
\hline $\begin{array}{l}\text { Thelypteris pilosa (M. Martens y Galeotti) Crawford } \\
\text { Woodsiaceae }\end{array}$ & Terrestre & 5,11 & & $\mathrm{X}$ & $\mathrm{X}$ & & & \\
\hline Cystopteris fragilis (L.) Bernh. & Terrestre & 5,6 & & & $\mathrm{X}$ & & & \\
\hline Woodsia mollis (Kaulf.) J. Sm. & Terrestre & 5,6 & & & $\mathrm{X}$ & & & \\
\hline
\end{tabular}

* Especies registradas por primera vez para el estado de Hidalgo.

Notholaena jacalensis Pray, P. polylepis (Roemer ex Kunze) T. Moore var. polylepis, P. guttata (Maxon) E. G. Andrews et Windham, P. madrensis (J. Sm.) A. R. Sm. et Tejero, Polypodium martensii Mett., S. extensa Underw. y S. ribae. Durante la revisión de las colecciones de helechos y licofitas en los herbarios revisados, se encontraron 3 especies del género Selaginella que no se recolectaron durante las visitas al municipio en estudio: Selaginella reflexa Underw., S. pallescens (C. Presl) Spring y $S$. extensa Underw.

En la figura 3 se observa el número de especies por rango altitudinal, y es el intervalo de $\operatorname{los} 2,101$ a 2,350 msnm el que

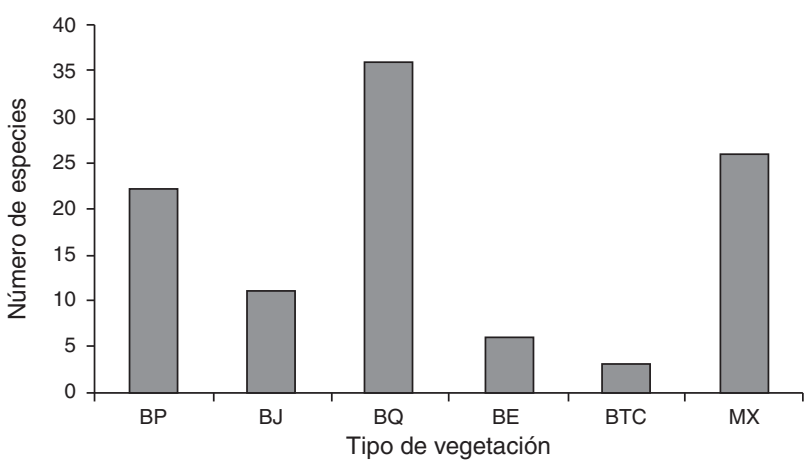

Figura 2. Riqueza de helechos y licofitas encontrada en cada tipo de vegetación presente en Cardonal. presenta la cifra mayor de taxones (45), pero en este confluyen diferentes tipos de vegetación. Mediante el análisis de correspondencia se obtuvo una gráfica (figura 4) que muestra que los tipos de vegetación BP, BQ y BTC presentan una composición florística de helechos y licofitas independiente, es decir, difieren notablemente entre sí y de BJ, BE y MX ubicándose en un cuadrante distinto en la gráfica. Asimismo, se observa que el BJ, BE y MX tienen una composición similar, agrupándose en el centro de la gráfica. Los factores 1 y 2 explican estos resultados con un valor de $54.64 \%$.

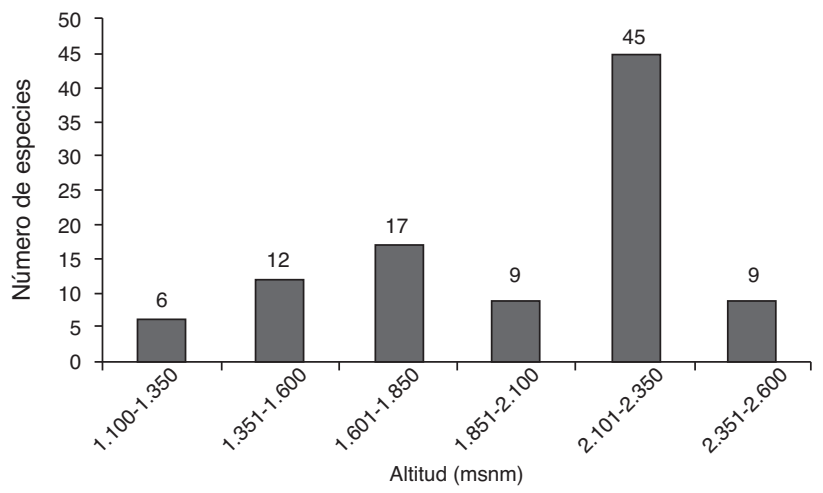

Figura 3. Riqueza de especies por rango altitudinal. 
Tabla 3

Número de especies por género.

\begin{tabular}{ll}
\hline Género & Número de especies \\
\hline Adiantum & 3 \\
Anemia & 2 \\
Argyrochosma & 1 \\
Asplenium & 4 \\
Astrolepis & 3 \\
Botrychium & 1 \\
Cystopteris & 1 \\
Cheilanthes & 5 \\
Elaphoglossum & 1 \\
Equisetum & 1 \\
Gaga & 1 \\
Llavea & 1 \\
Mildella & 1 \\
Myriopteris & 1 \\
Notholaena & 4 \\
Pecluma & 1 \\
Pellaea & 1 \\
Phlebodium & 1 \\
Pleopeltis & 1 \\
Polypodium & 1 \\
Pteridium & 1 \\
Pteris & 1 \\
Selaginella & 1 \\
Tectaria & 1 \\
Thelypteris & 1 \\
Woodsia & 1 \\
\hline & \\
\hline
\end{tabular}

Tabla 4

Número de familias, géneros y especies para cada tipo de vegetación.

\begin{tabular}{lllllll}
\hline Tipos de vegetación & BP & BJ & BQ & BE & BTC & MX \\
\hline Familias & 6 & 4 & 8 & 1 & 3 & 6 \\
Géneros & 11 & 7 & 19 & 4 & 3 & 11 \\
Especies & 21 & 11 & 36 & 6 & 3 & 26
\end{tabular}
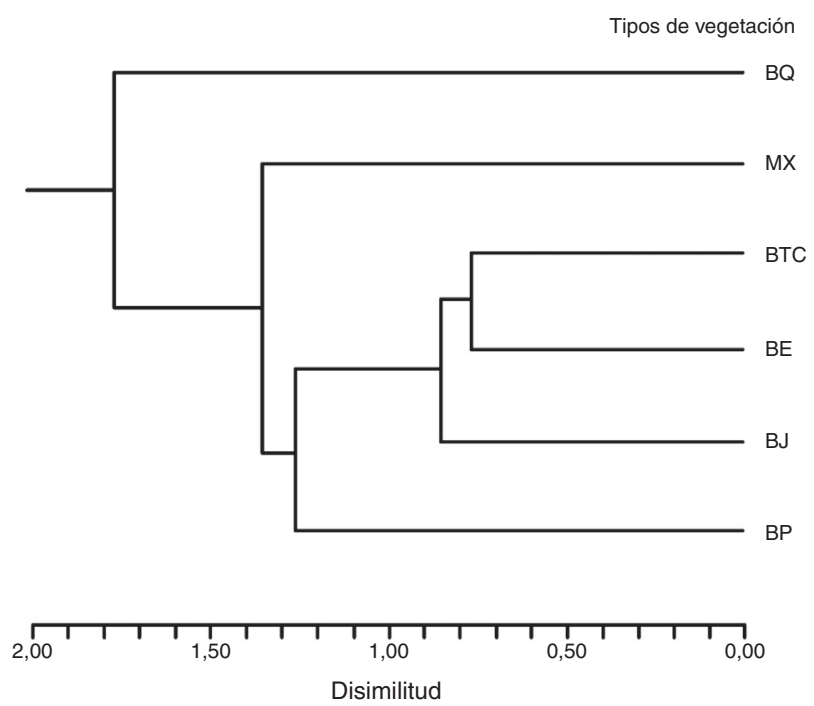

Figura 4. Relaciones entre los tipos de vegetación, utilizando un análisis de correspondencia.
Tipos de vegetación
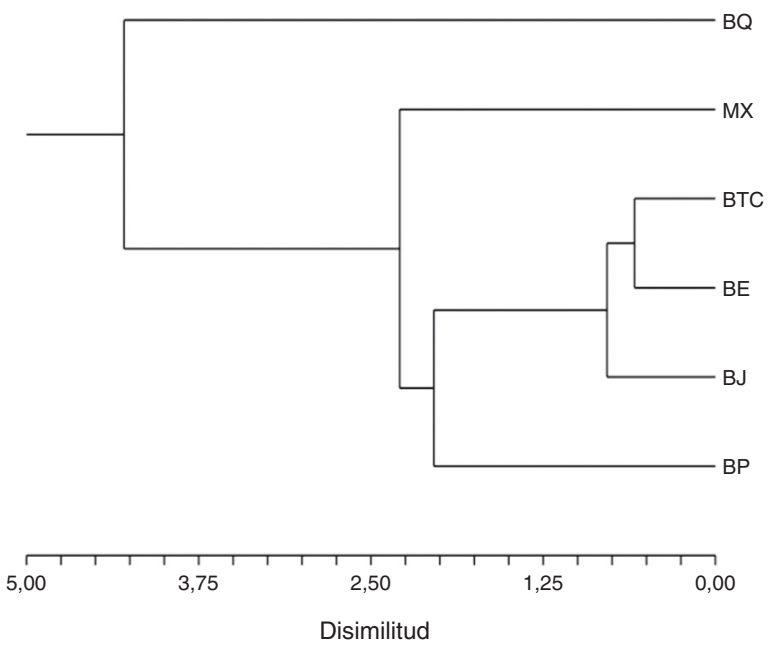

Figura 5. Análisis de agrupamiento mediante el método de varianza mínima de Ward.

El dendrograma obtenido mediante el método de varianza mínima de Ward (figura 5) muestra un solo grupo formado por BE, BTC y BJ, teniendo un valor de 0.79 unidades de disimilitud. Aunque BE y BTC son los sitios más parecidos (0.59 unidades), BJ se separa de ellos solo por 0.20 unidades, considerándose así que los 3 tipos de vegetación son similares en cuanto a número de especies; BP, BQ y MX cuentan un mayor número de taxones, siendo BQ el de mayor valor (tabla 4). El dendrograma obtenido mediante el análisis de grupos promedio (figura 6) muestra resultados similares, ya que las relaciones entre los tipos de vegetación son en general las mismas aun cuando los valores de disimilitud son menores.

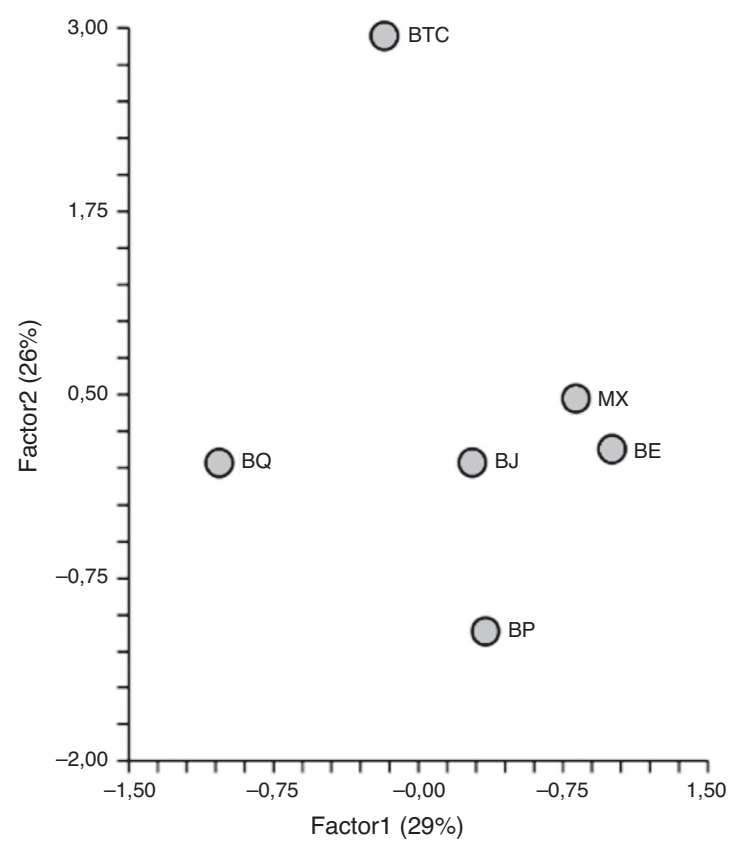

Figura 6. Análisis de agrupamiento mediante el método de grupos promedio. 


\section{Discusión}

El número de especies de helechos y licofitas encontradas en el municipio de Cardonal (63), es parecido al citado para el Parque Nacional los Mármoles (71) por Ramírez-Cruz et al. (2009), e incluso mayor al registrado por Ponce-Vargas et al. (2006) para el bosque mesófilo de montaña del municipio de Lolotla (32 especies) o el encontrado en Tenango de Doria (39) por Alcántara-Ayala y Luna-Vega (1997); y que el citado para la barranca de Metztitlán, en el plan de manejo de esta reserva de la biosfera (Conanp, 2003) solo se citan 4 especies de helechos y 2 de licofitas. El presente estudio indica que el municipio de Cardonal cuenta con una riqueza de helechos y licofitas importante y que sus condiciones climáticas y orográficas hacen posible la variedad de hábitats $\mathrm{y}$, por lo tanto, de especies.

En México se encuentran 186 especies endémicas de helechos y licofitas (Mickel y Smith, 2004). Algunos de los géneros con mayor porcentaje de endemismos, tales como Cheilanthes $(30 \%)$, Notholaena (42\%) y Selaginella $(35 \%)$ se encuentran en hábitats secos. Géneros como Asplenium y Polypodium que son predominantes en bosques húmedos tienen especies endémicas en regiones áridas (Mickel y Smith, 2004). En Cardonal se encontraron representados dichos géneros con 1 o 2 especies endémicas del país, de las cuales $N$. jacalensis es la especie con menor área de distribución, pues se cita solo de los estados de San Luis Potosí e Hidalgo (Mickel y Smith, 2004), dicha especie se encontró en el matorral xerófilo de la zona de estudio (tabla 2).

Los helechos y licofitas forman parte de la vegetación primaria o secundaria y en los límites de varios tipos de vegetación, muchas especies se distribuyen en diversos ecosistemas y otras que son característicos de ciertos hábitats (Riba, 1998). El bosque de pino (BP) se ubica en un rango altidudinal de 1,600 a 2,600 msnm, mientras que el bosque de Quercus (BQ) se localiza entre 2,200 y 2,400 msnm; sin embargo, en algunas localidades el bosque de pino se presenta en la ladera este de una montaña y el bosque de Quercus en la ladera oeste de la misma. Asimismo, el bosque tropical caducifolio (BTC) se encuentra entre 1,300 y $1,500 \mathrm{msnm}$, y en el mismo se encontraron 3 especies de helechos, de las cuales solo 1 es compartida con el bosque de pino y el bosque de Quercus. El matorral xerófilo (MX) se ubica entre 1,100 y 2,200 msnm con una pendiente muy pronunciada, y en el mismo se hallaron 6 especies de licofitas y 20 de helechos; en algunas localidades con MX se encontraban individuos aislados de Pinus, Quercus o Juniperus, lo cual permitió el establecimiento de especies epífitas como Pleopeltis polylepis var. polylepis y $P$. thyssanolepis. El bosque espinoso (BE) y el bosque de Juniperus (BJ) localizados entre 2,300 y $2,450 \mathrm{msnm}$, se relacionan en cuanto a composición florística de helechos con el matorral xerófilo, pues no se encontraron licofitas en estos hábitats. La relación se debe a que todas las especies de BE están presentes en el MX y de las 11 especies de helechos del BJ, 8 son compartidas con el MX. Cabe mencionar que en algunas localidades visitadas pertenecientes al matorral xerófilo (localidades19-21 en la figura 1) no se encontraron ni helechos ni licofitas, a pesar de visitar en varias ocasiones estos sitios.
Los análisis estadísticos muestran que de los 6 tipos de vegetación presentes en el municipio de Cardonal, el bosque de Quercus, el bosque tropical caducifolio y el bosque de pino son los 3 con mayor número de especies y, a su vez, con una composición florística de helechos y licofitas independiente, debido a las condiciones climáticas y fisiográficas presentes en dichas áreas, puesto que solo comparten 6 especies: Selaginella lepidophylla, Cheilanthes bonariensis, C. notholaenoides, Asplenium gentry, Pleopeltis polylepis var. polylepis y $P$. thyssanolepis.

A pesar de que la mayor parte del municipio está dedicado a la agricultura (Inegi, 2009), la diversidad de condiciones ambientales que se encuentran en Cardonal le confieren una importante riqueza florística en la zona con poca o nula perturbación, por lo que es importante considerar la conservación de dichos lugares.

\section{Agradecimientos}

A los curadores de las colecciones consultadas. A la Biól. Ana Alejandra Bautista-Cruz, la Biól. Claudia Verónica De la CruzMoreno y al Biól. Juan Carlos Rivera-Pacheco por su ayuda en el campo, así como a las autoridades del municipio de Cardonal, Hidalgo por el apoyo brindado para la realización de este estudio y a los revisores anónimos por sus comentarios y sugerencias.

\section{Referencias}

Alcántara-Ayala, O. y Luna-Vega, I. (1997). Florística y análisis biogeográfico del bosque mesófilo de montaña de Tenango de Doria, Hidalgo, México. Anales del Instituto de Biología. Universidad Nacional Autónoma de México, Serie Botánica, 68, 57-106.

Alcántara-Ayala, O. y Luna-Vega, I. (2001). Análisis florístico de dos áreas con bosque mesófilo de montaña en el estado de Hidalgo, México: Eloxochitlán y Tlahuelompa. Acta Botanica Mexicana, 54, 51-87.

Álvarez-Zúñiga, E., Sánchez-González, A., López-Mata, L. y Tejero-Díez, J. D. (2012). Composición y abundancia de las Pteridofitas en el bosque mesófilo de montaña del municipio de Tlanchinol, Hidalgo, México. Botanical Sciences, 90, 163-177.

Ceja-Romero, J., Mendoza-Ruiz, A., López-Ferrari, A. R., Espejo-Serna, A., Pérez-García, B. y García-Cruz, J. (2010). Las epífitas vasculares del estado de Hidalgo, México: diversidad y distribución. Acta Botanica Mexicana, 93, $1-39$.

Conanp (Comisión Nacional de Áreas Naturales Protegidas). (2003). Programa de manejo Reserva de la Biosfera Barranca de Metztitlán. México, D.F.: Comisión Nacional de Áreas Protegidas.

Espejo-Serna, A., López-Ferrari, A. R. y Salgado-Ugarte, I. (2004). A current estimate of angiosperm diversity in Mexico. Taxon, 53, 127-130.

Fay-Wei, L., Pryer, K. M. y Windham, M. D. (2012). Gaga, a new fern genus segregated from Cheilanthes (Pteridaceae). Systematic Botany, 37, 845-860.

Grusz, A. L. y Windham, M. D. (2013). Toward a monophyletic Cheilanthes: the resurrection and recircumscription of Myriopteris (Pteridaceae). PhytoKeys, 32, 49-64.

Inegi (Instituto Nacional de Estadística, Geografía e Informática). (2009). Prontuario de información geográfica municipal de los Estados Unidos Mexicanos. Cardonal, Hidalgo: Instituto Nacional de Estadística, Geografía e Informática.

Lorea, F. y Riba, R. (1990). Guía para la recolección y preparación de ejemplares para herbario de Pteridofitas. México, D.F.: Consejo Nacional de la Flora de México. A.C.

Mickel, J. T. y Smith, A. R. (2004). The Pteridophytes of Mexico. Memoirs of the New York Botanical Garden, 88, 1-1054. 
Pérez-Cervantes, A. (2009). Los helechos y licopodios del municipio de Calnali estado de Hidalgo, México. Tesis. Instituto de Ciencias Básicas e Ingeniería. Centro de Investigaciones Biológicas. Universidad Autónoma del Estado de Hidalgo.

Pérez-Paredes, M. G., Sánchez-González, A. y Tejero-Díez, J. D. (2012). Listado de licofitas y helechos del municipio de Zacualtipán de Ángeles, Hidalgo. México. Polibotánica, 33, 57-73.

Pérez-Paredes, M. G., Serrano, H., Álvarez, E. y Sánchez-González, A. (2011). Riqueza de especies de helechos y licopodios en el estado de Hidalgo. Herreriana, 7, 5-7.

Ponce-Vargas, A., Luna-Vega, I., Alcántara-Ayala, O. y Ruiz-Jiménez, C. A. (2006). Florística del bosque mesófilo de montaña de Monte Grande, Lolotla, Hidalgo, México. Revista Mexicana de Biodiversidad, 77, 177-190.

Ramírez-Cruz, S., Sánchez-González, A. y Tejero-Díez, J. D. (2009). La Pteridoflora del Parque Nacional Los Mármoles, Hidalgo, México. Boletín de la Sociedad Botánica de México, 84, 35-44.
Riba, R. (1998). Pteridofitas mexicanas: distribución y endemismo. En T. P. Ramammoorthy, R. Bye y A. Lot (Comps.), Diversidad biológica de México: orígenes y distribución (p. 369-385). México, D.F.: Instituto de Biología Universidad Nacional Autónoma de México.

Rzedowski, J. (1978). Vegetación de México. México: D.F.: Ed. Limusa.

Sánchez-Mejorada, H. y Chávez, C. (1951). Breves notas sobre las pteridofitas de la barranca de Omitlán, Hidalgo. Boletín de la Sociedad Botánica de México, 12, 28-36.

Serrano, M. H. (2010). Los helechos y licopodios del Parque Nacional El Chico. Tesis. Centro de Investigaciones Biológicas. Instituto de Ciencias Básicas e Ingeniería. Universidad Autónoma del Estado de Hidalgo.

Smith, A. R., Pryer, K. M., Schuettpelz, E., Korall, P., Schneider, H. y Wolf, P. G. (2006). A classification for extant ferns. Taxon, 55, 705-731.

Smith, A. R. y Tejero-Díez, J. D. (2014). Pleopeltis (Polypodiaceae), a redefinition of the genus and nomenclatural novelties. Botanical Sciences, 92, 43-58. 